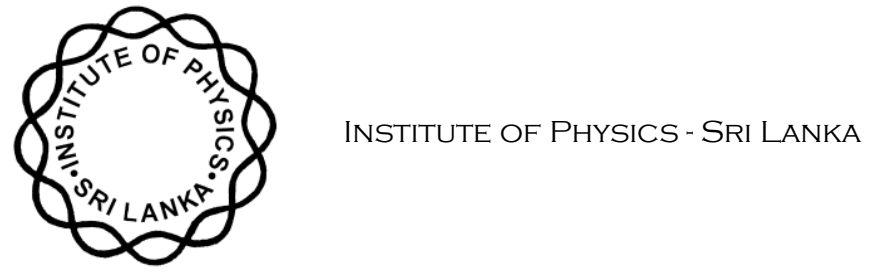

Research Article

\title{
Bound state solutions of the Klein - Gordon equation for deformed Hulthen potential with position dependent mass
}

\author{
Akaninyene D.Antia ${ }^{1}$, Akpan N.Ikot ${ }^{* 1}$, Eno E.Ituen ${ }^{1}$ and Ita O.Akpan² \\ ${ }^{I}$ Theoretical Physics Group,Department of Physics,University of Uyo-Nigeria. \\ ${ }^{2}$ Department of Physics, University of Calabar-Nigeria.
}

\begin{abstract}
We solve approximately the bound state solutions of the Klein - Gordon equation for deformed Hulthen potential with unequal scalar and vector potential for arbitrary $l$-state. We obtain explicitly the energy eigenvalues and the corresponding wave function expressed in terms of the Jacobi polynomials. We also discuss the energy eigenvalues of our result for three cases with equal and unequal scalar and vector potentials.
\end{abstract}

Keywords: Klein-Gordon equation; Hulthen potential; bound state

\section{INTRODUCTION}

In nuclear and high energy physics, one of the interesting problems is to obtain exact solution of the Klein - Gordon, Duffin - Kemmer - Petiau and Dirac equations for mixed vector and scalar potentials. When a particle is in a strong potential field, the relativistic effect must be considered, which gives the correction for non - relativistic quantum mechanics ${ }^{1}$. The Klein - Gordon, Dirac, and Duffin - Kemmer - Petiau wave equations are frequently used to describe the particle dynamics in relativistic quantum mechanics.

In relativistic quantum mechanics, one can apply the Klein - Gordon equation to the treatment of a zero-spin particle and the Dirac equation for spin half particle. In

\footnotetext{
*Corresponding Author E mail: ndemikot2005@yahoo.com
} 
recent years, many studies have been carried out to explore the relativistic energy eigenvalues and corresponding wave functions of the Klein-Gordon and Dirac equations $^{1-19}$.

These relativistic equations contain two objects: the four - vector linear momentum operation and the scalar rest mass. These allow one to introduce two types of potential coupling, which are the four vector potential $V(r)_{3}$ and the space - time scalar potential $S(r)$.

The Klein - Gordon equation with the vector and scalar potentials can be written as follows:

$$
\left[-\left(i \frac{\partial}{\partial t}-V(r)\right)^{2}-\nabla^{2}+(S(r)+M)^{2}\right] \Psi(r, \theta, \varphi)=0
$$

where $M$ is the rest mass, $i \frac{\partial}{\partial t}=E$ is the energy eigenvalues, $V(r)$ and $S(r)$ are the vector and scalar potentials respectively. For example, some authors have assumed that the scalar potential is equal to the vector potential and obtained the exact solutions of the Klein - Gordon equation and Dirac equation with some typical potential using different methods 5 . For example, these investigations have employed anharmonic oscillator potential $^{10}$, Kratzer potential ${ }^{11-12}$, ring shape pseudoharmonic potential ${ }^{13}$, Woods-Saxon potential $^{14}$, new ring-shaped non-spherical Harmonic oscillator ${ }^{15}$, Scarf potential ${ }^{16}$, Hartmann potential ${ }^{17}$, Poschl-Teller potential and Rosen Morse potential ${ }^{18}$. Different methods such as the Supersymmetry Quantum Mechanics ${ }^{19}$, Asymptotic iteration $\operatorname{method(AIM)})^{20}$ and Nikiforov-Uvarov(NU) $)^{14,21}$ and others have been used to solve the second order differential equations arising from these considerations.

However, the analytical solutions of the Klein - Gordon equations are possible only in the $\mathrm{s}-$ wave case with the angular momentum $l=0$ for some exponential type potential models $^{22-23}$. Conversely, when $l \neq 0$, one can only solve approximately the Klein - Gordon equation and the Dirac equation for some potential using a suitable approximation scheme ${ }^{24 .}$

The concept of the position dependent mass in the quantum mechanical systems has also attracted a lot of attention and research interest in recent times ${ }^{33}$. They are useful and have been applied in many fields, such as semiconductor physics ${ }^{25}$, quantum wells and quantum dots ${ }^{26}$, quantum liquids ${ }^{27}$ and semi conductor hetero-structures ${ }^{28}$ and others. Recently, the solutions of the non- relativistic wave equation with position dependent mass have been a line of great interest ${ }^{29-32}$ but there are only few contributions that give the solutions of the relativistic wave equation with position dependent mass in quantum mechanics ${ }^{33-36}$. One of us investigated the bound state 
solutions of the Klein-Gordon equation with Hulthen potential ${ }^{2}$ and being motivated by this success, we will attempt to solve the Klein-Gordon equation for deformed Hulthen potential with position dependent mass using the Nikiforov-Uvarov method.

In this paper, we attempt to solve approximately the arbitrary $l$-state Klein Gordon equation for the deformed Hulthen potential with an exponentially spatially dependent mass using the Nikiforov - Uvarov (NU) method. The centrifugal term in the Klein - Gordon equation is dealt with using a new improved approximation scheme ${ }^{37}$. The organization of this paper is as follows: In section 2, we review the NikiforovUvarov (NU) method brieftly. In section 3, Bound state solutions of Klein - Gordon equation is presented. In section 4 , solution of the radial equation of the Klein - Gordon equation via NU is presented. Conclusion is presented in section 5.

\section{REVIEW OF NIKIFOROV -UVAROV (NU) METHOD}

The NU method ${ }^{38}$ is based on solution of a general second-order linear differential equation with special orthogonal functions. In this method, for a given real or complex potential, the Schrödinger equation is transformed into a generalized equation of hypergeometric type with an approximate $s=s(r)$ coordinate transformation and it can be written in the following forms;

$$
\psi^{\omega}(s)+\frac{\bar{\tau}(s)}{\sigma(s)} \psi^{\prime}(s)+\frac{\bar{\sigma}(s)}{\sigma^{2}(s)} \psi(s)=0
$$

where $\sigma(s)$ and $\bar{\sigma}(s)$ are polynomials, at most second degree, and $\bar{\tau}(s)$ is a first degree polynomial. In order to find the exact solution to equation (2), we set the wave function as

$$
\psi(s)=\phi(s) \chi(s)
$$

and on substituting Eq. (3) into eq. (2) reduces Eq. (2) into hypergeometric type

$$
\sigma(s) \chi^{\circ}(s)+\tau(s) \chi^{\circ}(s)+\lambda \chi(s)=0
$$

where the wave function $\phi(s)$ is defined as a logarithmic derivative in the following forms and its solutions can be obtained from

$$
\frac{\phi(s)}{\phi(s)}=\frac{\pi(s)}{\sigma(s)}
$$

where $\pi(s)$ is at most first - degree polynomials.

The other part $\chi(s)$ is the hypergeometric type function whose polynomial solutions are given by the Rodrigues relation 


$$
\chi_{n}(s)=\frac{B_{n}}{\rho(s)} \frac{d^{n}}{d s^{n}}\left[\sigma^{n}(s) \rho(s)\right]
$$

where $B_{n}$ is the normalization constant and the weight function $\rho(s)$ must satisfy the condition and $\mathrm{n}$ is the order of the equation.

$$
\frac{d}{d s}(\sigma(s) \rho(s))=\tau(s) \rho(s)
$$

The function $\pi(s)$ and the parameter $\lambda$ required for this method are defined as follows:

$$
\begin{aligned}
& \pi(s)=\frac{\sigma^{\circ}-\bar{\tau}}{2} \pm \sqrt{\left(\frac{\sigma^{s}-\bar{\tau}}{2}\right)^{2}-\bar{\sigma}+K \sigma} \\
& \lambda=k+\pi(s)
\end{aligned}
$$

where the prime denotes differentiation.

On the other hand, in order to find the value of $k$, the expression under the square root must be square of a polynomial. Thus, a new eigenvalues equation becomes.

$$
\lambda=\lambda_{n}=-n \tau^{\circ}(s)-\frac{n(n-1)}{2} \sigma^{\varpi}(s), n=0,1,2, \ldots
$$

where

$$
\tau(s)=\bar{\tau}(s)+2 \pi(s)
$$

and its derivative is negative. By comparing eqs. (9) and (10), we obtain the energy eigen values.

\section{BOUND STATE SOLUTIONS OF KLEIN- GORDON EQUATION FOR DEFORMED HULTHEN POTENTIAL}

The three dimensional Klein- Gordon equation with the mixed vector and scalar potentials can be written as

$$
\left[\nabla^{2}+(V(r)-E)^{2}-(S(r)+M)^{2}\right] \psi\left(r_{s}, \theta\right)=0
$$

where $M$ is the rest mass, $E$ is the relativistic energy, $S(r)$ and $V(r)$ are the scalar and vector potentials respectively, $\nabla^{2}$ is the Laplace operator, c is the speed of light and $\hbar$ is the reduced Planck's constant which have been set to unity. In the spherical coordinates, the Klein - Gordon equation for a particle in the present of a deformed Hulthen potential 
$V(r)$ becomes

$$
\begin{aligned}
& {\left[\frac{1}{r^{2}} \frac{\partial}{\partial r}\left(r^{2} \frac{\partial}{\partial r}\right)+\left(\frac{1}{r^{2} \sin \theta} \frac{\partial}{\partial \theta}\left(\sin \theta \frac{\partial}{\partial \theta}\right)\right)+\frac{1}{r^{2} \sin ^{2} \theta} \frac{\partial^{2}}{\partial \varphi^{2}}\right.} \\
& \left.-2(E V(r)+M S(r))+V^{2}(r)-S^{2}(r)+E^{2}-M^{2}\right] \Psi\left(r_{y}, \theta, \varphi\right)=0
\end{aligned}
$$

If one assigns the corresponding spherical total wave function as

$$
\Psi\left(r_{s}, \varphi\right)=\frac{R(r)}{r} Y_{I m}(\theta, \varphi)
$$

where

$$
Y_{l m}\left(\theta_{v} \varphi\right)=\Theta(\theta) \phi(\varphi)
$$

then the wave equation (13) is separated into variables and the following equations are obtained.

$$
\begin{aligned}
& \frac{d^{2} R}{d r^{2}}+\left[E^{2}-M^{2}-2(E V(r)+M S(r))+V^{2}(r)-S^{2}(r)-\frac{\lambda}{r^{2}}\right] R(r)=0, \\
& \frac{d^{2} \Theta(\theta)}{d \theta^{2}}+\cot \theta \frac{d \Theta(\theta)}{d \theta}+\left[\lambda-\frac{m^{2}}{\sin ^{2} \theta}\right] \Theta(\theta)=0 \\
& \frac{d^{2} \Phi(\varphi)}{d \varphi^{2}}+m^{2} \Phi(\varphi)=0
\end{aligned}
$$

where $m^{2}$ and $\lambda=l(l+1)$ are the separation constants. The solutions for equation (16) and (17) are well known ${ }^{39}$.

\section{SOLUTIONS OF THE RADIAL EQUATION}

The deformed Hulthen scalar and vector potentials are respectively written as

$$
S(r)=\frac{S_{0} e^{-\operatorname{sEr}}}{\left(1-q e^{-2 W X}\right)}, V(r)=\frac{W_{0} e^{-2 W X}}{\left(1-q e^{-2 W X}\right)}
$$

where $S_{0^{y}} V_{0}$ are the potential depth, $q$ is the deformation parameter and $\alpha$ is an arbitrary constant. We define the position dependent mass in the form ${ }^{33}$

$$
M(r)=M_{0}\left[1-\frac{q e^{-\operatorname{sar}}}{\left(1-q e^{-\operatorname{Rar}}\right)}\right],
$$

where $M_{0}$ is the rest mass at equilibrium position. 
Substituting Eq. (18) and (19) into Eq. (15), we obtain the radial equation of Klein Gordon equation as

$$
\begin{aligned}
& \frac{d^{x} R(r)}{d r^{2}}+\left[E^{2}-M_{0}^{2}-\left(\left(M_{0} q+S_{0}\right)^{2}-V_{0}^{2}\right) \frac{e^{-4 W r}}{\left(1-q e^{-n Q r}\right)^{2}}\right. \\
& \left.+\left(2 M_{0}^{2} q+2 M_{0} S_{0}+E V_{0}\right) \frac{e^{-\operatorname{IQX}}}{\left(1-q e^{-2 W r}\right)}-\frac{R(R+1)}{r^{2}}\right] R(r)=0
\end{aligned}
$$

The centrifugal term in equation (20) can be evaluated using the new improved approximation scheme ${ }^{37}$.

$$
\frac{1}{r^{2}} \approx 4 \alpha^{2}\left[C_{0}+\frac{e^{-n a r}}{\left(1-q e^{-\operatorname{sar})^{2}}\right.}\right]
$$

where $C_{0}=\frac{1}{12}$ is an arbitrary dimensionless constant. In this study, we set $C_{0}=0$, which reduces Eq. (21) to conventional approximation scheme ${ }^{40}$. The choice of $C_{0}=0$, does not alter the physics of the problem under investigation.

Substituting Eq. (21) into Eq. (20), we obtain

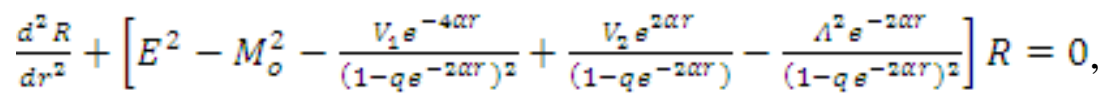

$$
\begin{aligned}
& \left.\begin{array}{c}
V_{1}=\left(M_{0} q+S_{0}\right)^{2}-V_{0}^{2} \\
\text { where } V_{2}=2 M_{0}^{2} q+2 M_{0} S_{0}+2 E V_{0} \\
A^{2}=4 \alpha^{2} l(l+1)
\end{array}\right\}
\end{aligned}
$$

Using a new variable $s=e^{-2 \alpha r}$ and substituting into Eq. (22), we have the following hypergeometric equation,

$$
\begin{aligned}
& \frac{d^{2} R(s)}{d s^{2}}+\frac{(1-q s)}{S(1-q s)} \frac{d R(s)}{d s}+\frac{1}{S(1-q s)}\left[-\left(\varepsilon^{2} q^{2}+\beta^{2}+\gamma^{2} q\right) s^{2}\right. \\
& \left.+\left(2 \varepsilon^{2} q+\gamma^{2}-\delta^{2}\right) s-\varepsilon^{2}\right] R(s)=0
\end{aligned}
$$

where the following dimensionless quantities have been used in obtaining equation (24):

$$
-\varepsilon^{2}=\frac{E^{2}-M_{0}^{2}}{4 \alpha^{2}}, \beta^{2}=\frac{\mathbb{V}_{1}}{4 \alpha^{2}}, \gamma^{2}=\frac{\mathbb{W}_{2}}{4 \alpha^{2}}, \delta^{2}=\frac{A^{2}}{4 \alpha^{2}}
$$

Now comparing Eq. (24) with Eq. (2), we get

$$
\begin{aligned}
& \sigma(s)=S(1-q s), \bar{\tau}(s)=1-q s, \\
& \bar{\sigma}(s)=-\left(\varepsilon^{2} q^{2}+\beta^{2}+\gamma^{2} q\right) s^{2}+\left(2 \varepsilon^{2} q+\gamma^{2}-\delta^{2}\right) s-\varepsilon^{2} .
\end{aligned}
$$


Substituting Eq. (26) into Eq. (8) gives

$$
\pi(s)=\frac{-q s}{2} \pm \frac{1}{2} \sqrt{\left(4 \varepsilon^{2} q^{2}+4 \beta^{2}+4 \gamma^{2} q+q^{2}-4 k q\right) s^{2}-4\left(\varepsilon^{2} q+\gamma^{2}-\delta^{2}-k\right) s+4 \varepsilon^{3}{ }_{s}}
$$

and we get two possible functions for each $k$ as

$$
\pi(s)=\frac{-q s}{2} \pm \frac{1}{2}\left\{\begin{array}{l}
\left(2 q \varepsilon-\sqrt{4 \delta^{2} q+4 \beta^{2}+q^{2}}\right) s-2 \varepsilon_{y} \text { for } k=\gamma^{2}-\delta^{2}+\varepsilon \sqrt{4 \delta^{2} q+4 \beta^{2}+q^{2}} \\
\left(2 q \varepsilon+\sqrt{4 \delta^{2} q+4 \beta^{2}+q^{2}}\right) s-2 \varepsilon_{y} \text { for } k=\gamma^{2}-\delta^{2}-\varepsilon \sqrt{4 \delta^{2} q+4 \beta^{2}+q^{2}}
\end{array}\right.
$$

For the polynomial of $\tau(s)=\bar{\tau}+2 \pi(s)$ which has a negative derivative, we get

$$
\begin{aligned}
& \pi(s)=\frac{-q s}{2}-\frac{1}{2}\left[\left(2 q \varepsilon+\sqrt{4 \delta^{2} q+4 \beta^{2}+q^{2}}\right) s-2 \varepsilon\right] \\
& k=\gamma^{2}-\delta^{2}-\varepsilon \sqrt{4 \delta^{2} q+4 \beta^{2}+q^{2}}
\end{aligned}
$$

Substituting Eqs. (26) and (29) into equation (11), we obtain

$$
\begin{aligned}
& \tau(s)=1-2 q(1+\varepsilon) s-\left(\sqrt{4 \delta^{2} q+4 \beta^{2}+q^{2}}\right) s+2 \varepsilon, \\
& \tau^{\prime}(s)=-2 q(1+\varepsilon)-\sqrt{4 \delta^{2} q+4 \beta^{2}+q^{2}}
\end{aligned}
$$

The parameter $\lambda=k+\pi^{v}(s)$ is obtained as

$$
\lambda=\gamma^{2}-\delta^{2}-\left(q+\sqrt{4 \delta^{2} q+4 \beta^{2}+q^{2}}\right) \varepsilon-\frac{-q}{2}-\frac{1}{2} \sqrt{4 \delta^{2} q+4 \beta^{2}+q^{2}}
$$

Substituting Eqs. (26) and (31) into Eq. (10), we obtain

$$
\lambda_{n}=2 n q(1+\varepsilon)+n \sqrt{4 \delta^{2} q+4 \beta^{2}+q^{2}}+n(n-1) q
$$

Comparing Eqs. (32) and (33), we have

$$
\varepsilon=\frac{\left[y^{2}-\delta^{2}-\left(n+\frac{1}{2}\right) \sqrt{\left.4 \delta^{2} q+4 \beta^{2}+q^{2}-q\left(n(n+1)+\frac{1}{2}\right)\right]}\right.}{2 q\left(n+\frac{1}{q}\right)+\sqrt{4 \delta^{2} q+4 \beta^{2}+q^{2}}}
$$

Substituting Eq. (25) into Eq. (34), we obtain

$$
E^{2}=M_{0}^{2}-4 \alpha^{2} \frac{\left[\frac{V_{n}}{4 \alpha^{2}}-\frac{A^{2}}{4 \alpha^{2}}-\left(n+\frac{1}{2}\right) \sqrt{\frac{A^{2} q^{2}}{\alpha^{2}}+\frac{V_{1}}{\alpha^{2}}+q^{2}}-q\left(n(n+1)+\frac{1}{n}\right)\right]^{2}}{\left(2 q\left(n+\frac{1}{2}\right)+\sqrt{\frac{M^{2} q^{2}}{\alpha^{2}}+\frac{V_{1}}{\alpha^{2}}+q^{2}}\right)^{2}}
$$


By using Eq. (23) in Eq. (35) the energy spectrum of the Klein - Gordon equation for the deformed Hulthen potential with position dependent mass is obtained as,

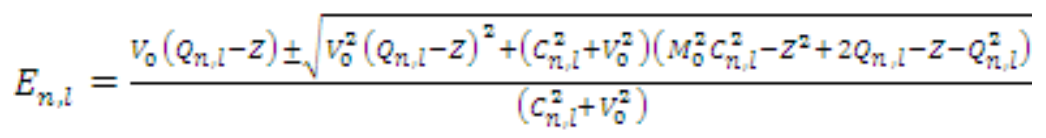

where

$$
\begin{gathered}
Q_{n, l}=2 \alpha\left[\left(n+\frac{1}{2}\right) \sqrt{4 \alpha^{2} l(l+1) q+\left(S_{0}+M_{0} q\right)^{2}-V_{0}^{2}+\alpha^{2} q^{2}}+\alpha l(l+1)+\right. \\
\left.\alpha\left(n(n+1)+\frac{1}{2}\right) q\right] \\
C_{n, l}=2 \alpha\left(n+\frac{1}{2}\right) q+\sqrt{4 \alpha^{2} l(l+1) q+\left(S_{0}+M_{0} q\right)^{2}-V_{0}^{2}+\alpha^{2} q^{2}}, \\
Z=M_{0}\left(S_{0}+M_{0} q\right)
\end{gathered}
$$

The corresponding wave function can now be calculated by first calculating the weight function $\rho(s)$. Using Eq. (7) the weight function is obtained as

$$
\rho(s)=(1-q s)^{v} S^{\mu}
$$

where $v=\frac{1}{q} \sqrt{4 \delta^{2} q+4 \beta^{2}+q^{2}}$ and $\mu=2 \varepsilon$.

Substituting Eq. (37) into the Rodrigues relation of Eq. (6), we get the eigen function $\chi_{m}(s)$ as

$$
\chi_{n}(s)=B_{n}(1-q s)^{-v} S^{-\mu} \frac{d^{n}}{d g^{n}}\left[(1-q s)^{n+v} s^{(n+\mu)}\right],
$$

where $B_{n}$ is the normalization constant.

The other part of the wave function in Eq. (3) is obtained by using $\frac{\phi^{s}(s)}{\phi(s)}=\frac{\pi(s)}{\sigma(s)}$ and solving the resulting differential equation yields,

$$
\phi(s)=(1-q s)^{\frac{1}{2}(1+v) s^{2}} s^{\frac{s}{2}}
$$

Combining equations (38) and (39), we obtain radial wave function as

$$
R(s)=A_{n}(1-s)^{\frac{1}{2}(1+v)} s^{\frac{\mu}{2}} P_{n}^{(\mu, v)}(s)
$$

where $A_{n}$ is the new normalization constant. The total wave function of the system is

$$
\psi\left(r_{s}, \theta\right)=\frac{A_{n}}{r}\left(1-e^{-2 \alpha r}\right)^{\frac{1}{2}(1+v)}\left(e^{-2 \alpha r}\right)^{\frac{\mu}{x}} P_{n}^{(\mu, v)}\left(1-e^{-2 \alpha r}\right) Y_{I}^{m}\left(\theta_{s} \varphi\right)
$$




\section{DISCUSSION}

Before we discussed the limiting cases of this potential, it is important to note that this potential under investigation is one of the short-range potential in physics and it plays a significant role in nuclear and molecular physics. The present potential can be written as

$$
V(r)=\frac{-\mathbb{V}_{0} e^{-a r r}}{1-e^{-a r}}
$$

Where we have mapped $V_{0} \rightarrow-Z e^{2} a_{y} a \rightarrow \frac{a}{2}, b \rightarrow a^{-1} q \rightarrow 1$ and $Z e^{2}$ is the strength of the potential, $a$ is the screening parameter and $b$ is the range of the potential. If we identify $\mathrm{Z}$ as the atomic number in this potential, one can use this potential to study the bound state of some diatomic molecules as reported in ref. [31].

A. Case I: $q=0, l \neq 0$

(i) For a constant mass case $q=0$, with $S_{0} \neq V_{0}$ the energy spectrum of Eq. (36)

becomes $E_{n, \mathbb{R}}=\frac{W_{0} W \pm \sqrt{W_{0}^{n} W^{n}+S_{0}^{n} N}}{S_{0}^{n}}$

where

$$
\begin{aligned}
W= & 2 \alpha\left[\left(n+\frac{1}{2}\right) \sqrt{S_{0}^{2}-V_{0}^{2}}+\alpha l(l+1)\right]-M_{0} S_{0} \\
N= & 4 \alpha M_{0} S_{0}\left[\left(n+\frac{1}{2}\right) \sqrt{S_{0}^{2}-V_{0}^{2}}+\alpha l(l+1)\right] \\
& -4 \alpha^{2}\left[\left(n+\frac{1}{2}\right)^{2}\left(S_{0}^{2}-V_{0}^{2}\right)-\alpha^{2} l^{2}(l+1)^{2}+2 \alpha(n+1) l(l+1) \sqrt{S_{0}^{2}-V_{0}^{2}}\right]-M_{0}^{2} V_{0}^{2} .
\end{aligned}
$$

In this circumstance if the vector potential is stronger than the scalar potential $V_{0}>S_{0}$, There is no bound state for the Klein-Gordon particles. However, if we use $S_{0}$ instead of $S_{0}+M_{0} q$ in Eq.(36a),then Eq.(36a) is the same as Eq.(42b). This implies that the mass function $M_{0} q$ only plays an additional scalar potential role. 
(ii) For $S_{0}=V_{0} \neq 0$

The energy spectrum for this case becomes,

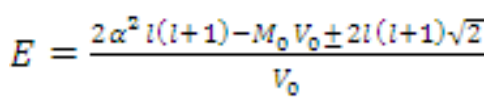

In this case there is a bound state for the Klein-Gordon particles.

B. $\quad$ Case II: $q \neq 0, l=0$

(i) If we consider the pure vector potential $\left(S_{0}=0, V_{0} \neq 0\right)$, the energy spectrum becomes

$$
E_{n}=\frac{\mathbb{W}_{0} D \pm \sqrt{W_{0}^{2} D^{2}+F G}}{F}
$$

where $D=2 \alpha\left(n+\frac{1}{2}\right) \sqrt{M_{0}^{2} q^{2} V_{0}^{2}+\alpha^{2} q^{2}}+2 \alpha^{2}\left(n(n+1)+\frac{1}{2}\right) q-M_{0}^{2} q$

$$
\begin{aligned}
& F=4 \alpha q\left(n+\frac{1}{2}\right)\left[\alpha\left(n+\frac{1}{2}\right) q+\sqrt{M_{0}^{2} q^{2} V_{0}^{2}+\alpha^{2} q^{2}}\right]+M_{0}^{2} q^{2}+\alpha^{2} q^{2} \\
& G=8 \alpha q\left(n+\frac{1}{2}\right) \sqrt{M_{0}^{2} q^{2} V_{0}^{2}+\alpha^{2} q^{2}}\left(M_{0}^{2}-\alpha^{2}\left(n(n+1)+\frac{1}{2}\right)\right) \\
& +4 \alpha^{2} q^{2}\left(M_{0}^{2}\left(n(n+1)+\frac{1}{2}\right)-\alpha^{2}\left(n(n+1)+\frac{1}{2}\right)^{2}\right) \\
& -4 \alpha^{2}\left(n+\frac{1}{2}\right)^{2}\left(M_{0}^{2} q^{2} V_{0}^{2}+\alpha^{2} q^{2}\right)+M_{0}^{2}\left(M_{0}^{2} q^{2} V_{0}^{2}+\alpha^{2} q^{2}\right) \\
& -M_{0}^{4} q^{2}+4 \alpha^{2} M_{0}^{2} q^{2}\left(n+\frac{1}{2}\right)^{2} .
\end{aligned}
$$

For these conditions there is always a bound states solution to the Klein-Gordon equation as long as $M_{0} q>V_{0}$ as reported in ref. [33]

(ii) For the $S_{0}=V_{0}$,

The bound state energy spectrum becomes,

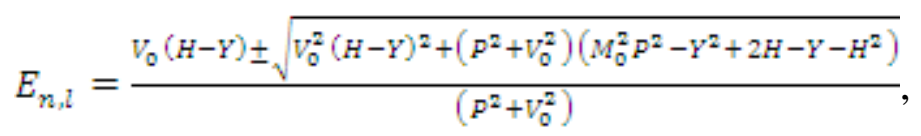


Where

$$
\begin{aligned}
& H=2 \alpha\left[\left(n+\frac{1}{2}\right) \sqrt{2 V_{0} M_{0} q+\left(M_{0} q\right)^{2}+\alpha^{2} q^{2}}+\alpha\left(n(n+1)+\frac{1}{2}\right) q\right] \\
& P=2 \alpha\left(n+\frac{1}{2}\right) q+\sqrt{2 V_{0} M_{0} q+\left(M_{0} q\right)^{2}+\alpha^{2} q^{2}}, \\
& Y=M_{0}\left(S_{0}+M_{0} q\right)
\end{aligned}
$$

Here for the s-wave with equal scalar and vector potential, the parameter $V_{1}$ in Eq. (23) will always have positive values and there are bound state solutions for the KleinGordon particles $^{41}$.

C. Case III: $q=0, l=0$

(i) If we consider $S_{0} \neq V_{0} \neq 0$, the eigen spectrum of the system becomes

$$
E_{n}=\frac{\mathbb{V}_{0} b^{J} \pm \sqrt{V_{0}^{n} \alpha^{y}+s_{0}^{n} c^{y}}}{s_{0}^{n}}
$$

where $a^{\prime}=4 \alpha^{2}\left(n+\frac{1}{2}\right)^{2}\left(S_{0}^{2}-V_{0}^{2}\right)+M_{0}^{2} S_{0}^{2}-4 \alpha\left(n+\frac{1}{2}\right) M_{0} S_{0} \sqrt{S_{0}^{2}-V_{0}^{2}}$

$$
\begin{aligned}
& b^{y}=2 a\left(n+\frac{1}{2}\right) \sqrt{S_{0}^{2}-V_{0}^{2}}-M_{0} S_{0} \\
& c^{y}=4 \alpha\left(n+\frac{1}{2}\right) M_{0} S_{0} \sqrt{S_{0}^{2}-V_{0}^{2}}-4 \alpha^{2}\left(n+\frac{1}{2}\right)^{2}\left(S_{0}^{2}-V_{0}^{2}\right)
\end{aligned}
$$

(ii) If we now consider the pure scalar potential $\left(S_{0} \neq 0, V_{0}=0\right)$, the energy spectrum of this system is obtained as

$$
E_{n}= \pm \sqrt{4 \alpha\left(n+\frac{1}{2}\right)\left[M_{0}-\alpha\left(n+\frac{1}{2}\right)\right]}
$$

By setting $\alpha=\frac{\alpha^{g}}{2}$, we obtain the energy levels as

$$
E_{n}= \pm \sqrt{\alpha^{\prime}\left(n+\frac{1}{2}\right)\left[2 M_{0}-\alpha^{s}\left(n+\frac{1}{2}\right)\right]}
$$

which is independent of the parameter $S_{\odot}$. This is in agreement with the results in the literatures ${ }^{33,41}$. Taking $\alpha^{y}=m_{\odot}=1$, the energy eigen values for this system is expressed as 


$$
E_{n}= \pm \sqrt{(n+1 / 2)\left(\frac{3}{2}-n\right)}
$$

which correspond to the bound state solutions for $n=0$ or 1 .

\section{CONCLUSION}

We investigated the bound state solution of the Klein-Gordon equation with unequal scalar and vector Hulthen potential for any arbitrary angular momentum quantum number $l$. We obtain the energy eigenvalues and the unnormalized wave function expressed in terms of the Jacobi polynomials. We have also discussed the limiting cases of the energy spectrum of our result which is in good agreement with those in the literature.

\section{ACKNOWLEDGEMENTS}

A.D.Antia dedicates this work to his dear wife for her love and care. A.N.Ikot acknowledges the Nandy-Research for their partial financial support. The authors wish to acknowledged the anonymous reviewer for his kind and positive suggestions which lead to the improvement of this manuscript.

\section{REFERENCES}

1. K.J. Oyewumi and C.O. Akoshile, Bound State solutions of the Dirac-Rosen-Morse potential with spin and pseudospin symmetry, Eur. Phys. J. A 45, (2010) 311-318.

2. A.N. Ikot, L.E.Akpabio and E.J. Uwah, Bound state solutions of the Klein-Gordon equation with the Hulthen potential, EJTP, $\underline{8}, 25$ (2011) 225-232.

3. G. Chen,Y.F. Diao and C.S. Jia, Bound states solutions of the Klein-Gordon equation with the generalized Poschl-Teller potential, Phys. Scr. 79 (2009) 065014.

4. F. Yasuk, A. Durmus and I. Boztosun, Exact analytical solution to the relativistic KleinGordon equation with noncentral equal scalar and vector potentials, J. Maths. Phys. 47 (2006) 082302.

5 A.N. Ikot, A.B. Udoimuk and L.E. Akpabio, Bound states solution of Klein-Gordon equation with typeI equal vector and scalar poschl-Teller potential for arbitrary l-state, Am. J. Sci. Ind. Res., 2 (2011) 179-183.

6. W.A. Yahya, K.J. Oyewumi, C.O. Akoshile and T.T. Ibrahim, Bound state solutions of the relativistic Dirac equation with equal scalar and vector Eckart potential using NikiforovUvarov method, JVR, $\underline{3}$ (2010) 27-34.

7. H. Akcay, C. Tezcan, Exact solutions of the Dirac equation with Harmonic oscillator Potential including a coulomb-like tensor potential, Int. J. Mod. Phys. C 20(6) (2009) 931940. 
8. A.D. Alhaidari, H.Bahlouli and A. Al-Hasan,. Dirac and Klein- Gordon equations with equal scalar and vector potentials, Phys. Lett. A $\underline{349}$, (2006) 87.

9. A.S. Dutra and G. Chen, On some classes of exactly-solvable Klein Gordon equations, Phys. Lett. A $\underline{349}$ (2006) 297.

10. G.F. Wei,C.Y. Long, Z. He,J.S. Qin, Bound state solution of the Dirac equation for a new anharmonic oscillator potential, Phys.Scripta,76 (2007) 442.

11. W.C. Qiang, Bound State of the Klein - Gordon and Dirac equations for potentials, Chin. Phys. 13 (2004) 575.

12. W.C. Qiang,.Bound states of the Klien - Gordon and Dirac equations for potential $V(r)=$ $A r^{-2}-B r^{-1}$, Chin. Phys. 12,10 (2003) 1054.

13 S. Ikhdair and R.Sever, Solution of the D-dimensional Klein Gordon equation with equal scalar and Vector ring - shaped pseudo harmonic potential, Int. J. Mod. Phys. C 19 (2008) 1425 .

14 C. Berkdemir, A. Berkdemir and R. Sever,. Systematical Approach to the Exact solution of the Dirac Equation for A special form of the Woods - Saxon potential, .Phys. A: Math. Gen. $\underline{399}$ (2006) 13455.

15. H. Xian - Quan, L. Guang, W. Zhi - Min, N. Lian - Bin and M. Yan, Solving Dirac Equation with new Ring - shaped Non - Spherical Harmonic oscillator potential, Commun. Theor. Phys. $\underline{53}$ (2010) $242-246$.

16. X.C.Zhang,Q.W.Liu,C.S.Jia and L.Z.Wang,Bound states of the Dirac equation into Vector and Scalar scarf -type potentials, Phys. Lett. A $\underline{340}$ (2005) 59.

17. A.de Souza Detra and M. Hott, Dirac equation exact solutions for generalized asymmetrical Hartmann Potentials, Phys. Lett. A $\underline{356}$ (2006) 215.

18. A.D. Alhaidari, Relativistic extension of shape - invariant potentials, J. Phys. A: Math. Gen. $\underline{34}$ (2001) 9827.

19 C.S. Jia, P. Gao and X.L. Peng, Exact solution of the Dirac - Eckart problem with spin and pseudospin symmetry, J. Phys. A: Math. Gen. $\underline{39}$ (2006) 7737.

20 H.Ciftci, R.L.Hall and N.Saad, Asymptotic iteration method for eigenvalue problems, J.Phys.A. 36, (2003) 11807.

21. Y.F. Cheng and T.O. Dai, Exact solution of the Klien - Gordon Equation with a Ring shape modified Kratzer potential, Chin. J. Phys. 45 (2007) 480 - 487.

22 Y.F. Diao,L.Z. Yi and C.S. Jia, Bound states of the Klien Gordon equation with Vector and Scalar five-parameter exponential - type potentials, Phys. Lett. A $\underline{32}$ (2004) 157.

23 X. Zou, L.Z. Yi and C.S. Jia, Bound states of the Dirac equation with Vector and Scalar Eckart potentials, Phys. Lett. A $\underline{346}$ (2005) 54.

24. Y. Xu, S. He and C.S. Jia, Approximate analytical solutions of the Klein - Gordon equation with the Poschl - Teller potential including the centrifugal term, Phys. Scripta $\underline{81}$ (2010) 045001.

25 G. Bastard, Wave Mechanics Applied to Semiconductor Heterostructures ( Les Edition de Physique,Les Ulis,1988).

26 L. Serra and E. Lipparini, Spin response of unpolarized quantum dots, Euro Phys. Lett. 40 (1997) 667.

27. F.A. de Saavedra, J. Boronat, A. Pollas and .Fabrocini, Effective mass of one ${ }^{4} \mathrm{He}$ atom in light ${ }^{3} \mathrm{He}$, Phys. Rev. B $\underline{50}$ (1994) 4248. 
28 S.Y. Yuen, Third order optical nonlinearity induced by effective mass gradient in

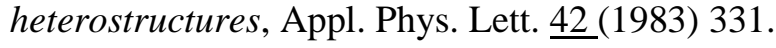

29 A.D. Alhaidari, Solutions of the nonrelativistic wave equation with position - dependent effective mass, Phys. Rev. A 66 (2002) 042116.

30 T. Tanaka, N. fold Supersymmetry in Quantum Systems with position - dependent mass, J. Phys. A: Math. Gen. $\underline{39}$ (2006) 219.

31. S.M. Ikhdair, Rotational and Vibration of diatomic molecule in the spatially - dependent mass Schrödinger equation with q-deformed Morse potential, Chem. Phys. 361 (2009) 9 17.

32. B. Roy and P. Roy, A Lie algebraic approach to effective mass Schrödinger equations, J. Phys. A: Math. Gen. 35 (2002) 3691.

33. T.Q.Dai, Bound state solutions of the $s$ - wave Klein - Gordon equation with position dependent mass for exponential potential, J. At. Mol. Sci., doi:10.4208/jams.012511.0305.030511a.

34 I.O. Vakarchuk, The Kepler problem in Dirac theory for a particle with position dependent mass, J. Phys. A: Math. Gen. $\underline{38}$ (2005) 4727.

35 A.D. Alhaidari, Solution of the Dirac equation with position - dependent mass in the coulomb field, Phys. Lett. A $\underline{322}$ (2004) 72.

36 S.M. Ikhdair, Exact Klein - Gordon equation with spatially dependent masses for unequal scalar - vector coulomb - like potentials, Eur. Phys. J.A 40 (2009) 143 - 149.

37 C.S. Jia, T. Chen and L.G. Cui, Approximate analytical solutions of the Dirac equation with the generalized Poschl - Teller potential including the pseudo - spin centrifugal term, Phys. Lett. A $\underline{373}$ (2009) 1621.

38. A.F. Nikiforov and V.B. Uvarov, Special Functions of Mathematical Physics (Basel, Birkhauser, 1988).

39 J. Sadeghi and B. Pourhassan, Exact solution of the Non- central modified Kratzer potential plus a Ring - shaped like potential by the factorization method, EJTP5 (2008) 193.

40 R.L. Greene and C. Aldrich, Variational wave functions for a screened coulomb potential, Phys. Rev. A 14 (1976) 2363.

41 F. Taskin, I. Boztosun and O. Bayrak, Exact solutions of Klein - Gordon equation with exponential scalar and vector potentials, Int. J. Theor. Phys.47 (2008) 1612. 